\title{
Prevalence and Risk Factors for Infection by Opisthorchis viverrini in an Urban Area of Mahasarakham Province, Northeast Thailand
}

\author{
Kusumaporn Chaiputcha ${ }^{1,3}$, Supannee Promthet ${ }^{1 *}$, Peter Bradshaw ${ }^{1}$
}

\begin{abstract}
The aim of this cross-sectional analytic study was to investigate the prevalence and risk factors for Opisthorchis viverrini $(\mathrm{OV})$ infection in an urban area of Northeastern Thailand. The participants were 254 household representatives aged 15 years or older living in the most urbanised part of Chiang Yuen municipality in Mahasarakham Province. All participants provided stool samples which were examined using the modified Kato-Katz procedure, and a structured interview questionnaire was used to collection demographic information, knowledge about $\mathrm{OV}$ infection, and the consumption of unsafely prepared freshwater fish. The data were analyzed using descriptive statistics and logistic regression. The overall prevalence of $\mathrm{OV}$ infection was $15.0 \%$, and in the multivariate analysis male gender was found to be significantly and positively associated with $\mathrm{OV}$ infection $\left(\mathrm{OR}_{\mathrm{adj}}=\mathbf{9 . 7 5}, 95 \% \mathrm{CI}\right.$ : 34.03-23.58) while education to secondary school level or above was a significant protective factor $\left(\mathrm{OR}_{\mathrm{adj}}=\mathbf{0 . 3 0}, \mathbf{9 5} \% \mathrm{CI}: \mathbf{0 . 1 2 - 0 . 7 4 )}\right.$. The eating of unsafely prepared fish and knowledge about $\mathrm{OV}$ were not significantly related to infection status. The findings were discussed in terms of issues for future research, especially the need to consider the possibility of higher rates of $\mathrm{OV}$ infection in urban areas than might be expected and to investigate the sources of infected fish products which may well be different from those in rural villages.
\end{abstract}

Keywords: Opisthorchis viverrini - prevalence-risk factors-Thailand

Asian Pac J Cancer Prev, 16 (10), 4173-4176

\section{Introduction}

Opisthorchis viverrini $(\mathrm{OV})$ is a food-borne parasite which is transmitted to people when they eat raw, undercooked or inadequately fermented cyprinoid fish infected with metacercariae, an intermediate form of the parasite (Saengsawang et al., 2012). While OV infection is not itself a serious health problem, it is major risk factor for the eventual development of cholangiocarcinoma, a malignancy of the bile ducts which is almost always fatal (Sripa et al, 2007; Shin et al., 2010; Songserm et al., 2012a; Sithithaworn et al., 2014).

Thailand has highest the prevalence of OV infection in the world, and the infection is particularly endemic in Northeastern Thailand where the rates of infection remained largely unchanged over the 10 year period preceding the last nationwide survey in 2009 when the reported prevalence in the region was $15.7 \%$. Rates varied from one northern province to another (4.6-60.8\%) and the rate for Mahasarakham Province, the location of the present study, was $11.6 \%$ (Sithithaworn et al., 2012).

The aim of this study was to investigate the prevalence and risk factors for $\mathrm{OV}$ infection in an urban area of Northeastern Thailand. Studies of this kind have tended to be conducted in rural villages rather than in urban environments because rural residents are assumed to be at higher risk of infection (Songserm et al 2012b). While there is some evidence that this may be the case (Kurathong et al., 1987; Wattanayingcharoenchai et al., 2011), further studies of OV infection in urban areas are needed. The findings of the present study were expected to be useful for planning OV infection control in the urban area chosen for this study.

\section{Materials and Methods}

This was a cross-sectional analytic study, which was conducted in the subdistrict municipality of Chiang Yuen in Mahasarakham Province, Northeastern Thailand, during the period January-April, 2014. The municipality has a population of 13,758 and includes 19 villages. Five villages in the centre of the municipality were chosen as the study area (total population 15 yrs and older: 4,912) because they represented the most urbanised part of the subdistrict.

\section{Subjects}

The sampling frame was the list of all registered 
Kusumaporn Chaiputcha et al

households in the five villages $(n=1,707)$, and 254 households were randomly selected by the proportionate stratified sampling of households in each village. A required sample size of 254 was estimated using formulae for proportion estimation (Lwanga and Lemeshow, 1991) and multiple logistic regression (Hsieh et al., 1998). The households were visited by a research assistant during the early morning or late afternoon to increase the inclusion of subjects who were at work for most of the day. One eligible member of each household was invited to participate in the study, provide his/her signed consent, and supply a stool sample. The eligibility criteria were: at least 15 years of age, residency in the subdistrict for the past 12 months, and ability to understand and respond to the study requirements. Where there was more one eligible subject available, the person was randomly chosen at the time of the visit. If no eligible subject was available, a neighbouring household was selected. All those invited to participate were willing to do so, but 12 failed to provide a stool sample and were replaced by a subject from the neighbouring household.

\section{Data collection}

All stool samples were analysed within 24 hours of collection for the presence of OV eggs using the modified Kato-Katz technique, and participants were interviewed by a research assistant using a structured questionnaire to obtain demographic information, assess knowledge about OV infection, and record the reported consumption 'yes' or 'no' of unsafely prepared fish in the past 12 months.
Table 1. Characteristics of Subjects

\begin{tabular}{|c|c|c|}
\hline Characteristics & Number $(n=254)$ & $\%$ \\
\hline \multicolumn{3}{|l|}{ Gender } \\
\hline Male & 104 & 40.9 \\
\hline Female & 150 & 59.1 \\
\hline \multicolumn{3}{|l|}{ Age (years) } \\
\hline $15-29$ & 25 & 9.8 \\
\hline $30-44$ & 60 & 23.6 \\
\hline $45-59$ & 116 & 45.7 \\
\hline $60+$ & 53 & 20.9 \\
\hline Mean (SD) & $48.6(13.7)$ & \\
\hline \multicolumn{3}{|l|}{ Marital status } \\
\hline Single & 29 & 11.4 \\
\hline Married & 186 & 73.2 \\
\hline Divorced/Widowed & 39 & 15.4 \\
\hline \multicolumn{3}{|l|}{ Education } \\
\hline No school & 1 & 0.4 \\
\hline Primary school & 166 & 65.4 \\
\hline Secondary school & 35 & 13.8 \\
\hline High school/college & 32 & 12.6 \\
\hline Diploma & 8 & 3.1 \\
\hline Graduate or higher & 12 & 4.7 \\
\hline \multicolumn{3}{|l|}{ Occupation } \\
\hline No paid employment/housewife & 32 & 12.6 \\
\hline Agriculture & 73 & 28.7 \\
\hline General labouring & 72 & 28.4 \\
\hline Commercial & 44 & 17.3 \\
\hline Students & 13 & 5.1 \\
\hline Government service & 14 & 5.5 \\
\hline Other & 6 & 2.4 \\
\hline
\end{tabular}

Table 2. Factors Associated with Opisthorchis viverrini (OV) Infection of Participants (n=254)

\begin{tabular}{|c|c|c|c|c|c|c|}
\hline \multirow[t]{2}{*}{ Factors } & \multirow{2}{*}{$\begin{array}{c}\text { Positive } \\
\text { OV } \\
\text { Number }(\%)\end{array}$} & \multirow{2}{*}{$\frac{\text { Negative }}{\text { OV }}$} & \multirow[t]{2}{*}{$\begin{array}{l}\text { ORcrude } \\
(95 \% \mathrm{CI})\end{array}$} & \multirow[t]{2}{*}{ p-value } & \multirow[t]{2}{*}{$\begin{array}{l}\text { ORadjusted } \\
(95 \% \mathrm{CI})\end{array}$} & \multirow[t]{2}{*}{ p-value } \\
\hline & & & & & & \\
\hline Gender & & & & $<0.001$ & & $<0.001$ \\
\hline Female & $7(4.7)$ & $143(95.3)$ & 1 & & 1 & \\
\hline Male & $31(29.8)$ & $73(70.2)$ & $8.67(3.64-20.65)$ & & $9.75(4.03-23.58)$ & \\
\hline Age (years) & & & & & 0.112 & \\
\hline $15-29$ & $1(4.0)$ & $24(96.0)$ & 1 & & & \\
\hline $30-44$ & $6(10.0)$ & $54(90.0)$ & $2.67(0.30-23.38)$ & & & \\
\hline $45-59$ & $22(19.0)$ & $94(81.0)$ & $5.62(0.72-43.79)$ & & & \\
\hline $60+$ & $9(17.0)$ & $44(83.0)$ & $4.91(0.59-41.10)$ & & & \\
\hline Education & & & & 0.020 & & 0.009 \\
\hline No school/primary school & $31(18.6)$ & $136(81.4)$ & 1 & & 1 & \\
\hline Secondary school or higher & $7(8.0)$ & $80(92.0)$ & $0.38(0.16-0.91)$ & & $0.30(0.12-0.74)$ & \\
\hline Occupation & & & & & 0.118 & \\
\hline No employment income /house wife & $2(6.3)$ & $30(93.8)$ & 1 & & & \\
\hline Agriculture & $17(23.3)$ & $56(76.7)$ & $4.55(0.98-21.04)$ & & & \\
\hline General labouring & $11(15.3)$ & $61(84.7)$ & $2.70(0.56-12.99)$ & & & \\
\hline Commercial & $5(11.4)$ & $39(88.6)$ & $1.92(0.35-10.61)$ & & & \\
\hline Student/government service/other & $3(9.1)$ & $30(90.9)$ & $1.5(0.23-19.63)$ & & & \\
\hline Monthly personal income (baht) & & & & 0.581 & & \\
\hline No income $/<5,000$ & $12(12.2)$ & $86(87.8)$ & 1 & & & \\
\hline $5,001-15,000$ & $24(17.0)$ & $117(83)$ & $1.47(0.69-3.10$ & & & \\
\hline$>15,001$ & $2(13.3)$ & $13(86.7)$ & $1.10(0.22-5.50)$ & & & \\
\hline Knowledge & & & & 0.107 & & \\
\hline Lower knowledge & $24(18.5)$ & $106(81.5)$ & 1 & & & \\
\hline Higher knowledge & $14(11.3)$ & $110(88.7)$ & $0.56(0.28-1.14)$ & & & \\
\hline Eating unsafely prepared fish & & & & 0.010 & & \\
\hline No & $1(2.8)$ & $35(97.2)$ & 1 & & & \\
\hline Yes & $37(17.0)$ & $181(83.0)$ & $7.15(0.95-53.88)$ & & & \\
\hline
\end{tabular}


Knowledge was assessed by responses to 18 five-level Likert-type statements with correct strong agreement scored as five points (maximum total knowledge score $=90)$. Half of the statements were negatively keyed so that disagreement was correct, and these items were reverse-scored. The areas of knowledge covered by these statements were the risks of OV infection (for example, "After treatment, people can be re-infected with liver flukes"), severity of the infection (for example, "Liver fluke infection can cause bile duct cancer") and the benefits of protection against infection (for example, "The best method of prevention is to stop eating raw fish"). Unsafely prepared fish was defined as one of five types of dishes containing raw freshwater fish and one containing freshwater fish which was likely to have been inadequately fermented. All participants found to be infected by OV were provided with a treatment dose of praziquantel.

\section{Statistical analysis and ethical approval}

Descriptive statistics were used to summarise the data. Higher and lower knowledge about OV infection was defined in terms of individual total knowledge scores being $\geq$ or $<$ the mean score for all participants, respectively. The associations between OV infection status and potential risk factors were analysed using unconditional logistic regression. Variables significant at the $p<0.25$ level in a univariate analysis were included in a multivariate analysis with backward elimination. Additional candidate variables were factors which were non-significant in the univariate analysis, but which have been reported as strong risk factors for OV infection in previous studies. Statistical analyses were performed using Stata version 10.0 (StataCorp LP, 2007). Statistical significance in the final model was set at $\mathrm{p} \leq 0.05$.

The research was approved by the Khon Kaen University Ethics Committee for Human Research (reference no. HE562264).

\section{Results}

The characteristics of the participating subjects are summarised in Table 1. Most (59.1\%) were female, and their mean age was 48.6 (SD:13.7) years. A large majority (65.8\%) had not received any formal education beyond primary school, and participants were most commonly employed in agriculture $(28.7 \%)$ or in some kind of general labouring activity $(28.4 \%)$. For those earning an income, this was less than 5,000 baht in almost a third $(32.7 \%)$ of the respondents.

In the multivariate analysis, male gender was found to be significantly and positively associated with infection $\left(\mathrm{OR}_{\mathrm{adj}}=9.75,95 \% \mathrm{CI}: 34.03-23.58\right)$, and education to secondary school level or above was a significant protective factor $\left(\mathrm{OR}_{\mathrm{adj}}=0.30,95 \% \mathrm{CI}: 0.12-0.74\right)$. The eating of unsafely prepared fish was a non-significant risk factor, and knowledge was unrelated to infection status

\section{Discussion}

The estimated prevalence of OV infection in an urban area of Mahasarakham was found to be no less than the latest reported prevalence for the province as whole. This was so even though the study area is well north of the many wetlands of Chi River basin which runs across the middle of the province.

The finding that male gender was significant risk factor for OV infection is consistent with results of another recent study in Northeastern Thailand (Thaewnongiew et al., 2014) and is supported by an earlier study (Sriamporn et al., 2004). While other surveys in Thailand have failed to show any statistically significant association between gender and infection (Rangsin et al., 2009; Kaewpitoon et al., 2012a; Kaewpitoon et al., 2012b; Saengsawang et al., 2012), all gender differences have indicated a higher prevalence in males. It is also notable that in present study the strength of the association with male gender was high: the odds of males being infected were almost 10 times higher than those for females. It is difficult to identify any particular reason for the inconsistency about the role of gender as a risk factor for OV infection, but the studies have used different sampling procedures and have focused on different target populations. Certainly, there appears to be a general belief that there is a predominantly male culture of eating raw and unsafely prepared freshwater fish (Grundy-Warr et al., 2012). This is an issue which requires further study to maximize the effectiveness of infection control campaigns.

The lack of association between knowledge about $\mathrm{OV}$ and infection status appears puzzling and needs to be examined further, especially because control programmes tend to rely heavily on educating the public about $\mathrm{OV}$ infection. There may be some similarities with smoking. Even though many smokers are aware that their habit is a serious health risk, they continue to smoke. One explanation for this is they rationalise the apparent inconsistencies between their knowledge and their behaviour by the adoption of functional beliefs (for example, "I enjoy smoking too much to give it up") and risk-minimising beliefs (for example, "Smoking is no more risky than lots of other things people do") (Fotuhi et al., 2013). By substituting 'eating raw freshwater fish' for 'smoking, it is not hard to see how knowledge can seem to become detached from behaviour, especially when that behaviour is strongly embedded in a social culture which probably frequently endorses such rationalizations. Whether or and to what extent this may be occurring is a matter for future research.

The inverse relationship between educational background and OV infection is consistent with one of the findings of Kaewpittoon et al. (2012a). In the present study the negative correlation does not seem to rely on better knowledge, and some alternative explanation may be necessary. One speculation is that the more highly educated people tend to be more socially mobile and are more likely to have moved away from their families of origin where there is still a strong cultural pressure to consume traditional dishes containing unsafely prepared fish. Again, this is an issue which can only be resolved in future studies.

The failure to demonstrate any positive association between infection status and the consumption of unsafely prepared fish, especially chopped raw fish in a spicey salad 
dressing (koi pla), is at variance with many other studies (for example, Rangsin et al., 2009; Suwannahitatorn et al., 2013; Thaewnongiew et al., 2014). This is particularly unexpected because the specific fish dishes explored were mainly those containing raw freshwater fish. There is a very possible methodological limitation which could explain this result: participants were asked to report on the consumption of fish dishes over the past 12 months. Accurate recall over such a long period of time for food consumed many times every day is most unlikely to be accurate. A further possible explanation is that the participants were not necessarily aware of the exact contents of the dishes and sauces which they were consuming. The authors suggest that there is increasing tendency for people, especially those living in urban areas, to buy foods and sauces from markets and roadside stalls without knowing the exact ingredients of their purchases. This is another topic for further investigation.

The findings of the present study indicate the need to consider the possibility of $\mathrm{OV}$ infection rates being higher than expected in urban areas and to investigate sources of infected fish products which may well be different from those in rural villages.

\section{Acknowledgements}

We are grateful for the Khon Kaen University Graduate Research Fund for Academic Year 2013, the National Research University Project of Thailand through the Center of Excellence in Specific Health Problems in the Greater Mekong Sub-region (SHeP-GMS), a research grant number NRU572001. The authors would like to thank all the staff at Chiang Yuen District Hospital and for their kind help. Thanks are also due to all participants in this study.

\section{References}

Fotuhi O, Fong GT, Mark P, et al (2013). Patterns of cognitive dissonance-reducing beliefs among smokers: a longitudinal analysis from the International Tobacco Control (ITC) Four Country Survey. Tob Control, 22, 52-8.

Grundy-Warr C, Andrews RH, Sithithaworn P, et al (2012). Raw attitudes, wetland cultures, life-cycles: Socio-cultural dynamics relating to Opisthorchis viverrini in the Mekong Basin. Parasitol Intern, 61, 65-70.

Hsieh FY, Bloch DA, Larsen MD (1998). A simple method of sample size calculation for linear and logistic regression. Stat Med, 17, 1623-34.

Kaewpitoon SJ, Rujirakul R, Ueng-arporn N, et al (2012a). Community-based cross-sectional study of carcinogenic human liver fluke in elderly from Surin Province, Thailand. Asian Pac J Cancer Prev, 13, 4285-8.

Kaewpitoon SJ, Rujirakul R, Kaewpitoon N (2012b). Prevalence of Opisthorchis viverrini infection in Nakhon Ratchasima Province, Northeast Thailand. Asian Pac J Cancer Prev, 13, 5245-9.

Kurathong S, Lerdverasirikul P, Wongpaitoon V, Pramoolsinsap C, Upatham ES (1987). Opisthorchis viverrini infection in rural and urban communities in northeast Thailand. Trans $R$ Soc Trop Med Hyg, 81, 411-4.

Lwanga SK, Lemeshow S (1991). Sample size determination in health studies: a practical manual. World Health
Organisation, Geneva.

Rangsin R, Mungthin M, Taamasri P, et al (2009). Incidence and risk factors of Opisthorchis viverrini infections in a rural community in Thailand. Am J Trop Med Hyg, 81, 152-5.

Saengsawang P, Promthet S, Bradshaw P (2012). Prevalence of OV infection in Yasothon Province, Northeast Thailand. Asian Pac J Cancer Prev , 13, 3399-402.

Saengsawang P, Promthet S, Bradshaw P (2013). Infection with Opisthorchis viverrini and use of praziquantel among a working-age population in Northeast Thailand. Asian Pac J Cancer Prev, 14, 2963-6.

Sithithaworn P, Andrews RH, Nguyen VD, et al (2012). The current status of opisthorchiasis and clonorchiasis in the Mekong Basin. Parasitol Intern, 61, 1-16.

Sithithaworn P, Yongvanit P, Duenngai K, Kiatsopit N, Pairojkul C (2014). Roles of liver fluke infection as risk factor for cholangiocarcinoma. J Hepatobiliary Pancreat Sci, 21, 301-8.

Shin H-R, Oh J-K, Masuyer E, et al (2010). Epidemiology of cholangiocarcinoma: An update focusing on risk factors. Cancer Sci, 101, 579-85.

Songserm N, Promthet S, Sithithaworn P, et al (2012a). Risk factors for cholangiocarcinoma in high-risk area of Thailand: Role of lifestyle, diet and methylenetetrahydrofolate reductase polymorphisms. Cancer Epidemiol, 36, 89-94.

Songserm N, Promthet S, Wiangnon S, Sithithaworn P (2012b). Prevalence and co-infection of intestinal parasites among Thai rural residents at high risk of developing cholangiocarcinoma. Asian Pac J Cancer Prev, 13, 6175-9.

Sriamporn S, Pisani P, Pipitgool V, et al (2004). Prevalence of Opisthorchis viverrini infection and incidence of cholangiocarcinoma in Khon Kaen, Northeast Thailand. Tropical Med Intern Health, 9, 588-94.

Sripa B, Kaewkes S, Sithithaworn P, et al (2007). Liver fluke induces cholangiocarcinoma. PLoS Med, 4, 201.

Sripa B, Kaewkes S, Intapan PM, Maleewong W, Brindley PJ (2010). Food-borne trematodiases in Southeast Asia: epidemiology, pathology, clinical manifestation and control. Advances Parasitol, 72, 305-50.

Sripa B, Brindley PJ, Mulvenna J, et al (2012). The tumorigenic liver fluke Opisthorchis viverrini multiple pathways to cancer. Trends Parasitol, 28, 395-407.

Sripa B, Thinkhamrop B, Mairiang E, et al (2012). Elevated plasma IL-6 associates with increased risk of advanced fibrosis and cholangiocarcinoma in individuals infected by Opisthorchis viverrini. PLoS Neglected Tropical Diseases, 6, 1-9.

StataCorp LP (2007). Stata Release 10: User's guide. College Station TX: Stata Press.

Suwannahitatorn P, Klomjit S, Naaglor T, et al (2013). A follow-up study of Opisthorchis viverrini infection after the implementation of control program in a rural community, central Thailand. Parasites Vectors, 6, 188.

Thaewnongiew K, Singthong S, Kutchamar S, et al (2014). Prevalence and risk factors for Opisthorchis viverrini infections in Upper Northeast Thailand. Asian Pac J Cancer Prev, 15, 6609-12.

Yongvanit P, Pinlaor S, Loilome W (2014). Risk biomarkers for assessment and chemoprevention of liver fluke-associated cholangiocarcinoma. J Hepatobiliary Pancreat Sci, 21, 309-15

Wattanayingcharoenchai S, Nithikathkul C, Wongsaroj T, et al (2011). Geographic information system of Opisthorchis viverrini in northeast Thailand. Asian Biomedicine, 5 , 687-91. 